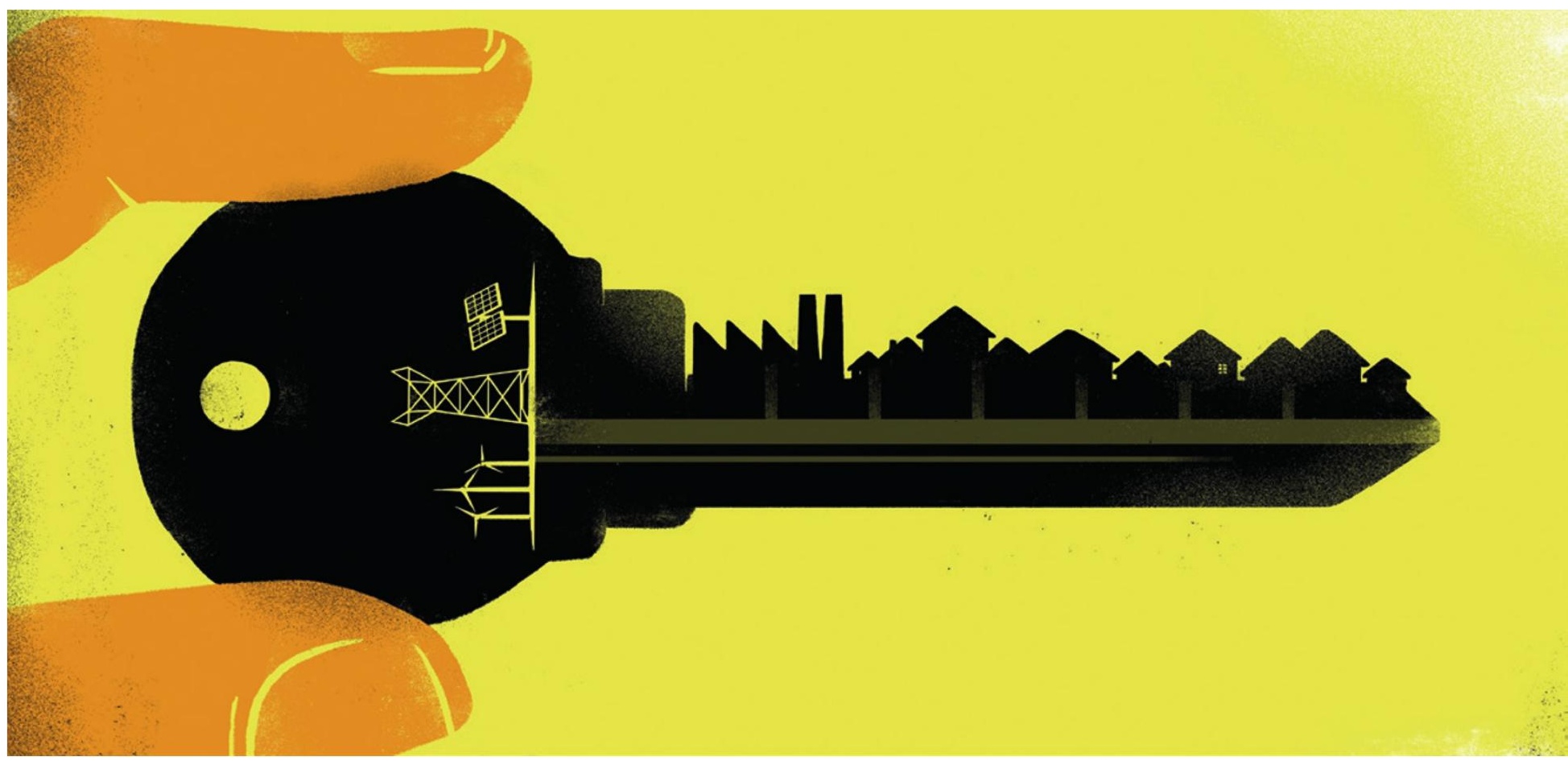

\title{
Power revolution
}

\section{Electrical grids increasingly depend on intermittent renewable sources. To smooth the supply out, utilities companies are testing alternatives to storing energy in conventional batteries.}

\section{BY PETER FAIRLEY}

I $\mathrm{t}$ is 2025 and another sweltering summer's day in California. Millions of solar panels are soaking up the Sun's rays to power the air-conditioning systems that keep homes and offices throughout the state cool. The devices are working efficiently thanks to an intelligent conversation taking place between the appliances and the electrical grid. As clouds drift across the Sun, casting shadows, the air conditioners deftly increase or decrease their output in sync with the varying flow of solar energy. In areas where the demand for electricity looks as though it will overload the power-transmission lines, home air-conditioning units take it in turns to go offline for an hour. In other areas, where solar power threatens to exceed demand, hot-water heaters are turned on to absorb the extra energy.

This imagined future power grid demonstrates the same degree of flexibility that energy-storage advocates predict will occur with the widespread implementation of batteries, but there is no electrochemistry involved - software manipulates energy-consuming equipment so that most electricity is used when it is most abundant, cheap or green.

The concept is called 'demand dispatch', because it would activate and deactivate power demand - much as grid operators dynamically dispatch electricity generated by power plants today. In the future, power grids will probably use both the 'virtual storage' created by demand dispatch and the true energy storage from batteries. But demand dispatch could be the bigger player of the two, with smart use of existing appliances offering a smaller environmental footprint and slimmer price tag than batteries.

"You can create flexibility much more cheaply by controlling electrical loads," says Sean Meyn, director of the Florida Institute for Sustainable Energy in Gainesville. "You do have to install communications equipment, but after that there's nothing to wear out, nothing to replace."

Meyn is not alone in anticipating that demand dispatch - rather than batteries will be the first line of defence for future grids (see 'Storage solutions'). A report published in May by the Massachusetts Institute of Technology Energy Initiative (go.nature.com/ogv7wa) said that controlling demand to maximize consumption of solar power as it is generated is cheaper than storing excess power in batteries. "Load shifting to hours when [solar] generation is high should be the first resource to look at," notes the report.

This non-battery option is already coming online as researchers and commercial developers establish communication protocols to link energy-consuming equipment to grid operators, develop algorithms for optimizing their deployment and experiment with the business models required to finance the systems. But demand dispatch also faces concerns about its security and its financial benefits. A major test in the US Supreme Court this October could unleash the potential of demand dispatch, or leave it languishing on the sidelines to be eclipsed by battery storage.

\section{REACTING TO DEMAND}

Utilities companies around the world must keep spare power plants sitting idle to serve energy-demand spikes that may occur on just a few days each year. To keep the number of extra plants to a minimum, they use 'demandside management' to help to control the need for power. This includes giving rebates for the 
use of efficient appliances or implementing time-of-day pricing that incentivizes energy use when grid demand is low. Many utilities firms also practise 'demand response', in which they pay selected power users - usually factories and other large industrial and commercial operations - to cut their power consumption when the power grids are really strained.

Demand dispatch to provide virtual energy storage is an advanced form of demand response, the growth potential of which is limited by its disruptive impact on power users shutting down a factory to save energy means lost revenue for the owners. Demand dispatch is about automating demand response and turning it into a more flexible, dynamic asset that customers hardly notice. The key is tapping into equipment that can be shut off for a few minutes or hours without inconveniencing users. Not all loads are a good fit, admits Mary Ann Piette, head of the Building Technology and Urban Systems Division at Lawrence Berkeley National Laboratory in California. Lighting is hard to switch off unobtrusively, for example.

But appropriate equipment is more common than you might think, she says. Electric water heaters, refrigerators and air-conditioning units can all be shut off for several hours at a time without affecting customers, thanks to thermal inertia, creating a window for virtual storage with little or no inconvenience.

Conventional demand response is exclusively about reducing load, but demand dispatch can often absorb electricity as well. Just as a battery can be charged and discharged, some electrical devices can be directed to preheat water, precool a space or bump up the pressure in a water line when strong winds and bright sunshine are providing surplus power. But aggregating many devices to create a substantial and reliable virtual-storage resource makes demand dispatch a more complex tool than batteries. "It's trickier," admits Robert Pratt, who leads the smart-grid programme at the Pacific Northwest National Laboratory in Richland, Washington. "But it may be cheaper."

Piette says that the system her group designed for commercial buildings costs US\$200-300 per kilowatt, considerably less than battery systems that can cost more than $\$ 1,000$ per kilowatt.

Meyn has studied the potential for controlling air-conditioning systems in commercial buildings to absorb the short-term minute-tominute variability in solar power. He says that many commercial air-conditioning systems could keep supply and demand in balance by adjusting their fan speeds depending on the availability of solar power. His studies at a building on the University of Florida campus suggest that this would have no detectable effect on comfort, and would add no cost to running the equipment. The grid impact, meanwhile, looks large. "We can accommodate a grid powered $50 \%$ by renewable energy without the use

of batteries," says Meyn.

Entrepreneurial firms are already beginning to make money by doing what Meyn proposes. ENBALA Power Networks in Vancouver, Canada, which has been selling grid-balancing services since 2011, taps into large power loads that can be controlled variably, such as industrial water pumps, and adjusts them in response to signals from grid operators to use the available energy more efficiently.

Several demonstration projects completed in the past three years have tested demand dispatch as a means of smoothing out larger energy swings over several hours, such as the large shifts in wind-power output caused by changing weather systems. PowerShift Atlantic, a demonstration completed this year by a consortium of power-grid operators in three of Canada's maritime provinces, choreographed 17 megawatts of equipment at 1,400 homes and businesses to absorb bursts and troughs in the wind power that, at certain times, generates more than half of the region's electricity.

Controlling $20-30 \%$ of power demand would be enough to almost flatten out the variability in the region's power supply, says Tom Osterhus, chief executive of Integral Analytics, the company in Cincinnati,

"We can
accommodate
a gridpowered
$50 \%$ by renewable
energy without
the use of
batteries."
Ohio, that made the control software for PowerShift Atlantic. Michel Losier, the project's manager and director of customer and community engagement for utility firm NB Power in New Brunswick, Canada, says that on a typical winter's day, when the outside air temperature is often below freezing, water tanks and electric heating account for about $55 \%$ of New Brunswick's peak load - exactly the kinds of appliances that could be controlled to shape demand.

\section{REMOTE CONTROL}

Researchers and entrepreneurs face several technical hurdles to turn projects like PowerShift Atlantic into the reality for power-grid operation. Tapping the virtual-storage potential of millions of devices is a massive communications and control challenge. And, because many of those devices reside in homes, it means dealing with residential power customers.

Forging a reliable communications link between residential power devices and grid operators emerged as a challenge in both PowerShift Atlantic and a series of recent smart-grid projects funded by the US Department of Energy. Utilities often struggled to get various systems to communicate with each other. Other projects found that communication using smart meters, many of which are optimized to exchange data with the utility every 15 minutes, was too slow for centralized dispatch of virtual storage.

As a result, operators are trying to bypass the smart meters and link to customers' home appliances using their broadband Internet connections. A standard protocol for Internet communications called OpenADR, which was developed by Piette's group, makes it easy for all the parts of the system to talk to each other. As the broad interconnection of consumer devices known as the Internet of Things progresses, costs will drop further and more loads will be able to participate, says Piette. "The future is rosy for virtual storage. Thanks to the Internet of Things, the cost of telemetry is getting cheaper."

But using the Internet comes with a caveat: it potentially leaves consumers and power grids vulnerable to cyberattacks and breaches in confidentiality. Pratt says that these risks will grow as connected appliances link to an ever-wider array of sites such as social media and onlineshopping outfits. "If your refrigerator is not only connected to the grid but also running through the Wild Wild West of the Internet, that may not be good," he says. "It's an emerging concern that in the Internet of Things your devices will be so promiscuous in who they talk to that we're really opening up Pandora's box."

Pratt, Meyn and others are working on control schemes that could reduce the cybersecurity threat by relying on more distributed intelligence, with decisions made by the devices themselves rather than a central grid computer. Meyn is developing a distributed control scheme that he hopes will ultimately require no more than one digital handshake per day, rather than constant communication between devices and the grid.

Then again, Internet connectivity also offers powerful opportunities, and Internet companies such as Google are keen to take advantage. Google acquired home-automation firm Nest Labs, based in Palo Alto, California, for $\$ 3.2$ billion in 2014 , and it is using it to build a virtual energy-storage system.

Nest's adaptive thermostats use sensors to guess when homes are unoccupied and then automatically put their heaters or air-conditioning units into a low-energy mode, making energy conservation easy. But they are also connected to the Internet, enabling the thermostat to factor local weather predictions into its behaviour. And, for homeowners who sign up to Nest's demand-dispatch programmes, connectivity creates a pool of potential virtual storage that utilities can access, through Nest, to dynamically adjust energy demand.

Nine utilities in the United States and Canada have signed up for Nest's demand-dispatch programmes, including Austin Energy in Texas, which had enrolled nearly 9,000 customers with smart thermostats by June. Together those homes can absorb or release up to 10.7 megawatts of power - a virtual storage capability that the utility expects to use 12-15 times per year to control demand spikes on hot days, 


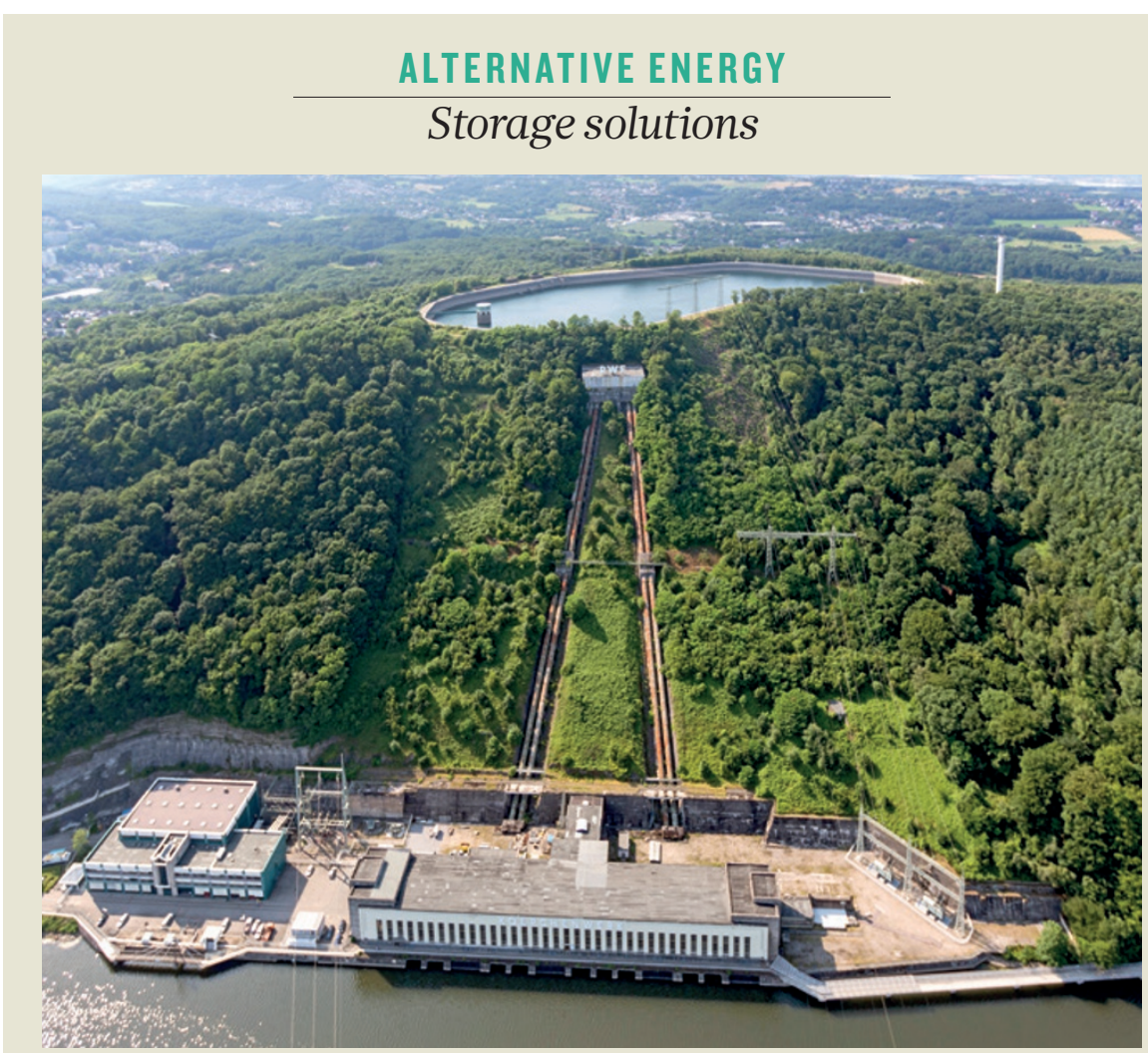

In pumped-storage hydropower stations, water is released to create energy when demand is high.

Grid-scale batteries face competition not only from 'virtual storage' systems that manipulate power demand, but also from other energy-storage technologies. Well-established alternatives include pumped storage hydropower, flywheels and compressed air.

Pumped-storage plants are the most affordable and proven means of largescale energy storage, and they account for $97.5 \%$ of energy-storage capacity installed on global power grids, according to the US Department of Energy. Most plants charge up by pushing water uphill using surplus energy generated overnight (when consumption is low); during peak load times, the water flows downhill and spins a turbine to generate power. Utilities are now eyeing pumped storage as a means of managing growing flows of renewable energy.

For example, the Kauai Island Utility Cooperative (KIUC), which runs the power grid for the Hawaiian island of Kauai, plans to spend between US $\$ 55$ million and $\$ 65$ million to repurpose disused sugar-plantation reservoirs to create a 25-megawatt pumped-storage plant to help it to manage solar power. The KIUC estimates that storing energy from solar panels for use at night will be roughly 35\% cheaper than running its oil-fired power plants. It will take time. The KIUC began studies in earnest in 2014, and says that it will be 2019 or later before the plant is built.
Flywheels, mechanical devices that are used to generate a power reserve, compete with batteries in applications that frequently require large power bursts for short periods, such as buffering power lines serving industrial mining equipment - heavy cycling conditions that degrade the capacity of batteries. Several recent flywheel projects target smaller power systems coping with high levels of intermittent renewable energy, using the flywheels to absorb and release power within microseconds to stabilize power demand in real time. In June, ABB in Zürich, Switzerland, installed a flywheel system on Alaska's Kodiak Island to help the local utility to cope with high levels of wind power. Darron Scott, president of the Kodiak Electric Association, says that the 2-megawatt flywheel replaced a 3-year-old lead-acid battery system that was wearing out faster than expected.

Another storage option is using electricity to compress air, which can then be converted back to electricity by using the air to spin a gas turbine. Early systems used underground caverns, including large installations in Alabama and in Germany. However, similar projects have been stymied by high equipment costs, energy losses and limited availability of appropriate geological formations. Morerecent efforts focus on portable, distributed systems that store compressed air in steel or composite tanks. P.F. when wholesale energy costs can rise from less than $\$ 50$ per megawatt-hour to more than $\$ 1,000$.

\section{POWER PITFALLS}

The biggest barrier to demand dispatch may be regulatory uncertainty over how it will be incorporated into the energy system, and how much grid operators should pay for it. There are positive signs for the market, as some states and regions forge ahead with rules that embrace demand dispatch, including PJM Interconnection, the US grid operator for 13 mid-Atlantic states and the District of Columbia.

Under US President Barack Obama, the Federal Energy Regulatory Commission (FERC) has enthusiastically embraced demand-side management. In 2011, FERC's Order 745 authorized wholesale power markets to pay companies that aggregate demand response the same price for managing megawatt-hours of demand as power-plant operators receive for generating energy. New York's Public Service Commission announced in June a mandate for all of the state's utilities to offer load-management programmes by mid- 2016 .

Demand response is also growing rapidly internationally. European countries are opening their energy markets to demand-response resources, and Japan and China are running pilot projects. Navigant Research, which specializes in clean-technology markets, predicts that annual global spending on demand response will grow from $\$ 2$ billion in 2015 to more than $\$ 12.8$ billion by 2023 as it expands beyond North America.

Despite these positive signs, there are still many energy markets in which regulators are taking a cautious approach, questioning whether the megawatt-hours of virtual storage that the technology promises will actually materialize when they are needed. This October, the US Supreme Court will decide whether to uphold a challenge - that compensation is a matter for state regulators - to FERC Order 745 by power generators. Advocates of demand dispatch, such as Meyn, say that the ruling is crucial for the technology's future.

Mei Shibata, co-founder of ThinkEco, a virtual-storage start-up in New York City, says that court challenges have already slowed growth in the United States by delaying the start of demonstration projects needed to convince energy regulators that the technology works. She predicts a big shakeout coming as venture-funded companies developing demand that can be dispatched run short of funds waiting for markets to mature. "The market will grow for residential and automated demand response, and convergence of demand response and the Internet of Things will happen, giving utilities better control over energy use," she says. "But how that happens isn't going to be a very pretty picture." -

Peter Fairley is a freelance science writer based in Victoria, British Columbia. 